\title{
THE QUANTUM ORBIT METHOD FOR GENERALIZED FLAG MANIFOLDS
}

\author{
JASPER V. STOKMAN
}

\begin{abstract}
Generalized flag manifolds endowed with the Bruhat-Poisson bracket are compact Poisson homogeneous spaces, whose decompositions in symplectic leaves coincide with their stratifications in Schubert cells. In this note it is proved that the irreducible *-representations of the corresponding quantized flag manifolds are also parametrized by their Schubert cells. An important step is the determination of suitable algebraic generators of the quantized flag manifolds. These algebraic generators can be naturally expressed in terms of quantum Plücker coordinates. This note complements the paper of the author and Dijkhuizen in Comm. Math. Phys. 203 (1999), 297-324, in which these results were established for a special subclass of generalized flag manifolds.
\end{abstract}

\section{Introduction}

The orbit principle of Kostant and Kirillov predicts a correspondence between the irreducible unitary representations of Lie groups and the coadjoint orbits of the underlying Lie algebra. As a natural generalization of this principle one expects a correspondence between the irreducible $*$-representations of quantized Poisson homogeneous spaces and their symplectic leaves. The key example is due to Soibel'man [6], who showed that the irreducible $*$-representations of the standard quantization $\mathbb{C}_{q}[U]$ of the regular functions on a compact, connected, simply connected, simple Lie group $U$ are parametrized by the symplectic leaves of $U$ (with the underlying Poisson structure on $U$ given by the Bruhat-Poisson bracket). In this note the correspondence is further investigated for generalized flag manifolds, which form a substantial subclass of Poisson $U$-homogeneous spaces.

A generalized flag manifold $G / P$, with $G$ the complexification of $U$ and $P \subseteq$ $G$ a parabolic subgroup, can be viewed as a real $U$-homogeneous space $U / K$, with $K \subseteq U$ isomorphic to a compact real form of the Levi factor of $P$. The flag manifold $U / K$ is a Poisson $U$-homogeneous space with symplectic foliation naturally parametrized by the Schubert cells of $G / P$. The quantization $\mathbb{C}_{q}[U / K]$ of $U / K$ can be realized as a subalgebra of $\mathbb{C}_{q}[U]$, defined in terms of invariance properties with respect to a suitable quantum subgroup $\mathbb{C}_{q}[K] \subseteq \mathbb{C}_{q}[U]$.

It is proved in [8] that the equivalence classes of the irreducible $*$-representations of $\mathbb{C}_{q}[U / K]$ are naturally parametrized by Schubert cells, provided that

Received June 5, 2002.

2000 Mathematics Subject Classification. $20 \mathrm{G} 42$. 
$\mathbb{C}_{q}[U / K]$ is algebraically generated by certain explicit products of quantum Plücker coordinates. This algebraic assumption was verified case by case for special examples of flag manifolds $U / K$, in particular when $U / K$ is a Hermitean symmetric space. In this note this algebraic assumption is proved for arbitrary flag manifold $U / K$ using an analogue of the Stone-Weierstrass Theorem for type I $C^{*}$-algebras. At the end of the note we extend these results to Poisson $U$ homogeneous spaces $U / K^{0}$, where $K^{0}$ is the semisimple part of $K$.

\section{Plücker coordinates on quantized flag manifolds}

In this section we compare several different quantized function algebras on generalized flag manifolds. We start by introducing the necessary notations and definitions.

2.1. Structure theory. Let $\mathfrak{g}$ be a complex simple Lie algebra, $\mathfrak{h} \subset \mathfrak{g}$ a Cartan subalgebra and $R \subset \mathfrak{h}^{*}$ the corresponding root system. Let $\Sigma=\left\{\alpha_{1}, \ldots, \alpha_{r}\right\}$ be a fixed choice of (ordered) simple roots and denote $R^{+} \subset R$ for the corresponding set of positive roots. Denote $\mathfrak{b}_{ \pm}$for the Borel subalgebras

$$
\mathfrak{b}_{ \pm}=\mathfrak{h} \oplus \bigoplus_{\alpha \in \pm R^{+}} \mathfrak{g}_{\alpha}
$$

where $\mathfrak{g}_{\alpha}$ is the root space corresponding to the root $\alpha$. We denote $(\cdot, \cdot)$ for the inner product on $\mathfrak{h}^{*}$ dual to the Killing form on $\mathfrak{g}$, and $W$ for the Weyl group of $R$. We use the short hand notation $s_{i} \in W$ for the reflection corresponding to the simple root $\alpha_{i}$.

Let $G$ be the connected, simply connected, real analytic Lie group with Lie algebra $\mathfrak{g}$. Let $B_{ \pm} \subset G$ be the Borel subgroups corresponding to the Lie algebras $\mathfrak{b}_{ \pm}$. The standard parabolic subgroups $P_{S}$ of $G$ containing $B_{+}$are naturally parametrized by subsets $S \subseteq \Sigma$, or equivalently, by parabolic sub root systems $R_{S}=R \cap \operatorname{span}\{S\} \subseteq R$. The Levi factor of the Lie algebra $\mathfrak{p}_{S}=\operatorname{Lie}\left(P_{S}\right)$ is given by

$$
\mathfrak{l}_{S}=\mathfrak{h} \oplus \bigoplus_{\alpha \in R_{S}} \mathfrak{g}_{\alpha}
$$

Using the identification of $\mathfrak{h}$ with its dual space $\mathfrak{h}^{*}$ via the Killing form, we define $H_{\alpha} \in \mathfrak{h}$ to be the Cartan element associated to the coroot $d_{\alpha}^{-1} \alpha \in \mathfrak{h}^{*}$ $(\alpha \in R)$, where $d_{\alpha}=(\alpha, \alpha) / 2$. The real span of the $H_{\alpha}$ 's $(\alpha \in R)$ is a real form $\mathfrak{h}_{0}$ of the Cartan subalgebra $\mathfrak{h}$. A compact real form $\mathfrak{u}$ of $\mathfrak{g}$ can now be chosen in such a way that $\mathfrak{k}_{S}:=\mathfrak{p}_{S} \cap \mathfrak{u}=\mathfrak{l}_{S} \cap \mathfrak{u}$ is a compact real form of $\mathfrak{l}_{S}$ for any subset $S \subseteq \Sigma$. Explicitly, $\mathfrak{u}$ is defined by

$$
\mathfrak{u}=i \mathfrak{h}_{0} \oplus \bigoplus_{\alpha \in R^{+}} \mathbb{R}\left(E_{\alpha}-E_{-\alpha}\right) \oplus \bigoplus_{\alpha \in R^{+}} \mathbb{R} i\left(E_{\alpha}+E_{-\alpha}\right),
$$

with $E_{\alpha} \in \mathfrak{g}_{\alpha}$ root vectors satisfying $\left[E_{\alpha}, E_{-\alpha}\right]=H_{\alpha}, \kappa\left(E_{\alpha}, E_{-\alpha}\right)=d_{\alpha}^{-1}$ and $\left[E_{\alpha}, E_{\beta}\right]=c_{\alpha, \beta} E_{\alpha+\beta}$ with $c_{\alpha, \beta} \in \mathbb{R}$ whenever $\alpha+\beta \in R$. Let $U \subset G$ be the 
connected Lie subgroup with Lie algebra $\mathfrak{u}$. The Lie subgroup $U \subset G$ is closed, compact and simply connected.

We now shortly recall the Bruhat-Poisson structure on $U$ (see e.g. [4] and references therein for basic facts on Poisson-Lie groups). Define a real Lie subalgebra $\mathfrak{b} \subset \mathfrak{g}$ by

$$
\mathfrak{b}=\mathfrak{h}_{0} \oplus \bigoplus_{\alpha \in R^{+}} \mathfrak{g}_{\alpha}
$$

Then $(\mathfrak{g}, \mathfrak{u}, \mathfrak{b})$ is a Manin triple with respect to the imaginary part of the Killing form. Thus $\mathfrak{u}$ inherets the structure of a Lie bialgebra. The associated PoissonLie group structure on $U$ is called the Bruhat-Poisson structure. Poisson-Lie subgroups of $U$ with respect to the Bruhat-Poisson bracket on $U$ can be classified as follows. Denote $\mathfrak{k}_{S}^{0}=\left[\mathfrak{k}_{S}, \mathfrak{k}_{S}\right]$ for the semisimple part of the reductive Lie algebra $\mathfrak{k}_{S}$.

Proposition 2.1. Let $K \subseteq U$ be a connected Lie subgroup with Lie algebra $\mathfrak{k}$. Then $K \subseteq U$ is a Poisson-Lie subgroup with respect to the Bruhat-Poisson bracket on $U$ if and only if $\mathfrak{k}_{S}^{0} \subseteq \mathfrak{k} \subseteq \mathfrak{k}_{S}$ for some subset $S \subseteq \Sigma$.

Proof. First observe that any real subspace $\mathfrak{k} \subseteq \mathfrak{u}$ satisfying $\mathfrak{k}_{S}^{0} \subseteq \mathfrak{k} \subseteq \mathfrak{k}_{S}$ is a Lie subalgebra of $\mathfrak{u}$ since $\mathfrak{k}_{S}=\mathfrak{k}_{S}^{0} \oplus Z\left(\mathfrak{k}_{S}\right)$ with $Z\left(\mathfrak{k}_{S}\right)$ the center of the Lie algebra $\mathfrak{k}_{S}$.

A connected Lie subgroup $K \subseteq U$ is a Poisson-Lie subgroup if and only if the orthocomplement $\mathfrak{k}^{\perp} \subseteq \mathfrak{b}$ of its Lie algebra $\mathfrak{k}$ in $\mathfrak{b}$ is an ideal (here the orthocomplement is taken with respect to the imaginary part of the Killing form). This class of Lie subalgebras $\mathfrak{k} \subseteq \mathfrak{u}$ is in one-to-one correspondence with real Lie subalgebras $\mathfrak{p} \subseteq \mathfrak{g}$ containing $\mathfrak{b}$. The correspondence is given by $\mathfrak{k}=\mathfrak{p} \cap \mathfrak{u}$ and $\mathfrak{p}=\mathfrak{k} \oplus \mathfrak{b}$. The real linear spaces

$$
\mathfrak{p}=\xi \oplus \bigoplus_{\alpha \in R^{+} \cup R_{S}} \mathfrak{g}_{\alpha}
$$

with $S \subseteq \Sigma$ and with real linear spaces $\xi$ satisfying

$$
i \mathfrak{h}_{0, S} \oplus \mathfrak{h}_{0} \subseteq \xi \subseteq \mathfrak{h}, \quad \mathfrak{h}_{0, S}=\mathbb{R}-\operatorname{span}\left\{H_{\alpha} \mid \alpha \in R_{S}\right\},
$$

are all the possible real Lie subalgebras $\mathfrak{p} \subseteq \mathfrak{g}$ containing $\mathfrak{b}$ (compare with the classification of the complex parabolic Lie subalgebras of $\mathfrak{g}$ ). The proposition follows by intersecting these Lie subalgebras $\mathfrak{p}$ with the compact real form $\mathfrak{u}$.

For a given subset $S \subseteq \Sigma$ we write $K_{S} \subseteq U$ (respectively $K_{S}^{0} \subseteq U$ ) for the connected Lie subgroup with Lie algebra $\mathfrak{k}_{S}$ (respectively $\mathfrak{k}_{S}^{0}$ ). Both $K_{S} \subseteq U$ and $K_{S}^{0} \subseteq U$ are closed Poisson-Lie subgroups of $U$. The homogeneous spaces $U / K_{S}$ and $U / K_{S}^{0}$ inheret a natural Poisson structure from $U$ (which will also be called the Bruhat-Poisson structure). In this note we study the quantization of the Poisson $U$-homogeneous space $U / K_{S}$ in detail. In Section 4 we formulate the main results for the quantization of the Poisson $U$-homogeneous space $U / K_{S}^{0}$.

Observe that $U / K_{S}$ is isomorphic to the generalized flag manifold $G / P_{S}$ as a real $U$-homogeneous space, since $K_{S}=P_{S} \cap U$ and $U$ acts transitively on $G / P_{S}$. 
The symplectic foliation of $U / K_{S} \simeq G / P_{S}$ coincides with the Schubert cell decomposition of $G / P_{S}$. The Schubert cells of $G / P_{S}$ are parametrized by the coset space $W / W_{S}$, where $W_{S} \subseteq W$ is the parabolic subgroup generated by the simple reflections $\left\{s_{i} \mid i: \alpha_{i} \in S\right\}$. The minimal coset representatives

$$
W^{S}=\left\{w \in W \mid l\left(w s_{\alpha}\right)>l(w), \quad \forall \alpha \in S\right\}
$$

with $l(w)$ the length of the Weyl group element $w \in W$, form a complete set of representatives for $W / W_{S}$. They satisfy

$$
l(u v)=l(u)+l(v), \quad \forall u \in W^{S}, \forall v \in W_{S} .
$$

2.2. Quantum groups. Fix $0<q<1$ and denote $U_{q}(\mathfrak{g})$ for the Drinfel'dJimbo quantized universal enveloping algebra over $\mathbb{C}$. The algebraic generators are denoted by $K_{i}^{ \pm 1}$ and $X_{i}^{ \pm}(i=1, \ldots, r)$, where the $K_{i}^{ \pm 1}$ correspond to Cartan elements and the $X_{i}^{+}$(respectively $X_{i}^{-}$) correspond to the root vectors of $\mathfrak{g}$ for the simple roots $\alpha_{i}$ (respectively $-\alpha_{i}$ ). For the explicit commutation relations we refer to $[8,(3.2)]$. We denote $U_{q}(\mathfrak{h})$ for the unital, commutative subalgebra of $U_{q}(\mathfrak{g})$ generated by the $K_{i}^{ \pm 1}(i=1, \ldots, r)$. Recall that $U_{q}(\mathfrak{g})$ is a Hopf- $*$-algebra, with the usual formulas for the counit $\epsilon$, comultiplication $\Delta$ and antipode $S$ (see $[8,(3.3)]$ ). Our present choice of $*$-structure on $U_{q}(\mathfrak{g})$ reflects the choice of compact real form $\mathfrak{u}$ of $\mathfrak{g}$; on the generators of $U_{q}(\mathfrak{g})$ it is explicitly defined by

$$
\left(K_{i}^{ \pm 1}\right)^{*}=K_{i}^{ \pm 1}, \quad\left(X_{i}^{+}\right)^{*}=q_{i}^{-1} X_{i}^{-} K_{i}, \quad\left(X_{i}^{-}\right)^{*}=q_{i} K_{i}^{-1} X_{i}^{+}
$$

with $q_{i}=q^{d_{i}}$ and $d_{i}=d_{\alpha_{i}}=\left(\alpha_{i}, \alpha_{i}\right) / 2$.

Let $P$ be the weight lattice of the root system $R$. Let $P^{+}$be the cone of dominant weights and $\left\{\varpi_{1}, \ldots, \varpi_{r}\right\}$ the fundamental weights with respect to the fixed choice of (ordered) simple roots $\Sigma=\left\{\alpha_{1}, \ldots, \alpha_{r}\right\}$. Denote $V(\lambda)$ for the irreducible finite dimensional $U_{q}(\mathfrak{g})$-module with highest weight $\lambda \in P^{+}$. The weight decomposition of $V(\lambda)$ is written as

$$
\begin{aligned}
V(\lambda) & =\bigoplus_{\mu \leq \lambda} V(\lambda)_{\mu}, \\
V(\lambda)_{\mu} & =\left\{v \in V(\lambda) \mid K_{i} \cdot v=q^{\left(\mu, \alpha_{i}\right)} v, \forall i\right\},
\end{aligned}
$$

where $\leq$ is the dominance order on $P$ with respect to the positive roots $R^{+}$. We fix a highest weight vector $0 \neq v_{\lambda} \in V(\lambda)_{\lambda}$ for each $\lambda \in P^{+}$once and for all.

The quantized function algebra $\mathbb{C}_{q}[U]$ is the Hopf-*-subalgebra of the Hopf-*algebra dual of $U_{q}(\mathfrak{g})$, spanned by the matrix coefficients of the finite dimensional $P$-weighted $U_{q}(\mathfrak{g})$-representations (see e.g. [8, (3.5)] for the definitions of the Hopf- $*$-algebra maps on $\left.\mathbb{C}_{q}[U]\right)$. The analogue of the Peter-Weyl Theorem is the decomposition

$$
\mathbb{C}_{q}[U]=\bigoplus_{\lambda \in P^{+}} W(\lambda)
$$


with $W(\lambda)$ the span of the matrix coefficients of $V(\lambda)$. Note that $\mathbb{C}_{q}[U]$ is an $U_{q}(\mathfrak{g})$-bimodule with respect to the left and right regular actions

$$
(X \cdot a)(Y)=a(Y X), \quad(a \cdot X)(Y)=a(X Y),
$$

where $a \in \mathbb{C}_{q}[U]$ and $X, Y \in U_{q}(\mathfrak{g})$. The Peter-Weyl decomposition (2.1) then coincides with the decomposition of $\mathbb{C}_{q}[U]$ in simple $U_{q}(\mathfrak{g})$-bimodules.

2.3. Quantized flag manifolds and Plücker coordinates. In the remainder of this note we fix an arbitrary subset $S \subseteq \Sigma$ of the simple roots. We identify the fixed subset $S=\left\{\alpha_{i_{1}}, \ldots, \alpha_{i_{l}}\right\}$ of $\Sigma$ with the corresponding subset $\left\{i_{1}, \ldots, i_{l}\right\}$ of $\{1, \ldots, r\}$. We furthermore fix a dominant weight $\Lambda \in P^{+}$which is supported on $\Sigma \backslash S$, and regular dominant with respect to $\Sigma \backslash S$. In other words, $\Lambda$ is of the form $\Lambda=\sum_{i=1}^{r} m_{i} \varpi_{i}$ with $m_{i}=0$ for $i \in S$ and $m_{i}>0$ for $i \in \Sigma \backslash S$.

Let $\widetilde{V}(\Lambda)$ be the finite dimensional irreducible $G$-representation with highest weight $\Lambda$ and fix a highest weight vector $0 \neq \widetilde{v}_{\Lambda} \in \widetilde{V}(\Lambda)_{\Lambda}$. Let $\mathbb{C}[G]$ be the algebra of regular functions on $G$. Let $F_{\Lambda}$ be the $G$-orbit of the line $\mathbb{C} \widetilde{v}_{\Lambda}$ in the projective space $\mathbb{P}(\widetilde{V}(\Lambda))$. Then $F_{\Lambda}$ is naturally isomorphic to $G / P_{S}$ as a complex projective variety. The algebra $\mathcal{F}_{\Lambda}$ of regular functions on the affine cone over $F_{\Lambda}$ is the unital subalgebra of $\mathbb{C}[G]$ generated by the matrix coefficients $\widetilde{f}_{\Lambda}=$ $f\left(\cdot \widetilde{v}_{\Lambda}\right),\left(f \in \widetilde{V}(\Lambda)^{*}\right)$. In this note we regard $\mathcal{F}_{\Lambda}$ as the algebra of holomorphic polynomials on (the affine cone over) $F_{\Lambda}$.

The algebra of antiholomorpic polynomials on $F_{\Lambda}$ is defined as follows. Let $0 \neq \xi_{\Lambda} \in\left(\widetilde{V}(\Lambda)^{*}\right)_{-\Lambda}$ be a lowest weight vector of the $G$-representation $\widetilde{V}(\Lambda)^{*}$ dual to $\widetilde{V}(\Lambda)$. Then the $G$-orbit of the line $\mathbb{C} \xi_{\Lambda}$ in the projective space $\mathbb{P}\left(\widetilde{V}(\Lambda)^{*}\right)$ is naturally isomorphic to $G / P_{S}^{o p p}$, where $P_{S}^{o p p}$ is the parabolic subgroup of $G$ associated to $S \subseteq \Sigma$ which contains the opposite Borel subgroup $B_{-}$. We call the unital subalgebra $\overline{\mathcal{F}}_{\Lambda}$ of $\mathbb{C}[G]$ generated by the regular functions

$$
G \ni g \mapsto \xi_{\Lambda}\left(g^{-1} \cdot v\right), \quad(v \in \tilde{V}(\Lambda)),
$$

the algebra of antiholomorphic polynomials on $F_{\Lambda}$.

We have the following natural quantum analogues, cf. Soibel'man [7]. Let $V(\lambda)^{*}$ be the linear dual of $V(\lambda)$. For $f \in V(\lambda)^{*}$ we denote $f_{\lambda} \in W(\lambda) \subset \mathbb{C}_{q}[U]$ for the matrix coefficient

$$
f_{\lambda}(X)=f\left(X \cdot v_{\lambda}\right), \quad \forall X \in U_{q}(\mathfrak{g}) .
$$

\section{Definition 2.2.}

(a) The unital subalgebra $\mathcal{F}_{\Lambda}^{q} \subseteq \mathbb{C}_{q}[U]$ generated by $f_{\Lambda}\left(f \in V(\Lambda)^{*}\right)$ is the quantum algebra of holomorphic polynomials on $F_{\Lambda}$.

(b) The unital subalgebra $\overline{\mathcal{F}}_{\Lambda}^{q} \subseteq \mathbb{C}_{q}[U]$ generated by $f_{\Lambda}^{*}\left(f \in V(\Lambda)^{*}\right)$ is the quantum algebra of antiholomorphic polynomials on $F_{\Lambda}$.

Let $\mathcal{B}$ be a basis of $V(\Lambda)^{*}$ which is compatible with the weight decomposition of $V(\Lambda)$, in the sense that each $b \in \mathcal{B}$ is supported on only one weight space $V(\Lambda)_{\mu}, \mu=\mu(b) \in P$. Then the matrix coefficients $f_{\Lambda}(f \in \mathcal{B})$ are called holomorphic quantum Plücker coordinates on $F_{\Lambda}$. They algebraically generate 
the algebra $\mathcal{F}_{\Lambda}^{q}$. The corresponding images $f_{\Lambda}^{*}$ under the $*$-involution are called the antiholomorphic quantum Plücker coordinates on $F_{\Lambda}$. They algebraically generate the algebra $\overline{\mathcal{F}}_{\Lambda}^{q}$.

We next define two closely related $*$-algebras $\mathbb{C}_{q}\left[U / K_{S}\right]$ and $\mathbb{A}_{\Lambda}$. Let $U_{q}\left(\mathfrak{k}_{S}\right) \subseteq$ $U_{q}(\mathfrak{g})$ be the unital Hopf-*-subalgebra algebraically generated by $K_{i}^{ \pm 1}(i=$ $1, \ldots, r)$ and by $X_{j}^{ \pm}(j \in S)$.

\section{Definition 2.3.}

(a) The $*$-algebra of quantized regular functions on the Poisson $U$-homogeneous space $U / K_{S}$ is defined by

$$
\mathbb{C}_{q}\left[U / K_{S}\right]=\left\{a \in \mathbb{C}_{q}[U] \mid X \cdot a=\epsilon(X) a, \forall X \in U_{q}\left(\mathfrak{k}_{S}\right)\right\} .
$$

(b) Let $\mathbb{A}_{\Lambda}$ be the unital $*$-subalgebra of $\mathbb{C}_{q}[U]$ algebraically generated by the elements $f_{\Lambda} \cdot g_{\Lambda}^{*} \in \mathbb{C}_{q}[U]$ for all $f, g \in V(\Lambda)^{*}$.

Remark 2.4. The elements $f_{\Lambda} \cdot g_{\Lambda}^{*} \in \mathbb{C}_{q}[U]\left(f, g \in V(\Lambda)^{*}\right)$ are explicitly given by

$$
\left(f_{\Lambda} \cdot g_{\Lambda}^{*}\right)(X)=\sum f_{\Lambda}\left(X_{(1)}\right) \overline{g_{\Lambda}\left(S\left(X_{(2)}\right)^{*}\right)}=\sum f\left(X_{(1)} \cdot v_{\Lambda}\right) \overline{g\left(S\left(X_{(2)}\right)^{*} \cdot v_{\Lambda}\right)}
$$

for $X \in U_{q}(\mathfrak{g})$, where $\Delta(X)=\sum X_{(1)} \otimes X_{(2)}$.

The *-subalgebra $\mathbb{C}_{q}\left[U / K_{S}\right] \subset \mathbb{C}_{q}[U]$ can alternatively be defined in terms of invariance properties with respect to a natural quantum subgroup $\mathbb{C}_{q}\left[K_{S}\right] \subseteq$ $\mathbb{C}_{q}[U]$ (see $[8,(4.4)]$ ). Observe that both $\mathbb{A}_{\Lambda}$ and $\mathbb{C}_{q}\left[U / K_{S}\right]$ are invariant under the right regular $U_{q}(\mathfrak{g})$-action.

We next discuss the connections between the algebras $\mathcal{F}_{q}, \overline{\mathcal{F}}_{q}, \mathbb{A}_{\Lambda}$ and $\mathbb{C}_{q}\left[U / K_{S}\right]$. It is instructive to consider the classical setup $(q=1)$ first. Let $\chi_{\Lambda}\left(\right.$ respectively $\left.\chi_{\Lambda}^{*}\right)$ be the character of $P_{S}$ (respectively $P_{S}^{o p p}$ ) defined by

$$
p \cdot \widetilde{v}_{\Lambda}=\chi_{\Lambda}(p) \widetilde{v}_{\Lambda}, \quad p^{\prime} \cdot \xi_{\Lambda}=\chi_{\Lambda}^{*}\left(p^{\prime}\right) \xi_{\Lambda}
$$

for $p \in P_{S}$ and $p^{\prime} \in P_{S}^{o p p}$. The classical analogues of the holomorphic (respectively antiholomorphic) Plücker coordinates are in the space $\Gamma\left(G \times{ }_{P_{S}} \mathbb{C}_{\chi_{\Lambda}}\right)$ (respectively $\Gamma\left(G \times{ }_{P_{S}^{\text {opp }}} \mathbb{C}_{\chi_{\Lambda}^{*}}\right)$ ) of sections of the line bundle $G \times{ }_{P_{S}} \mathbb{C}_{\chi_{\Lambda}}$ (respectively $\left.G \times{ }_{P_{S}^{\text {opp }}} \mathbb{C}_{\chi_{\Lambda}^{*}}\right)$. Observe that $\mathbb{C}_{\chi_{\Lambda}} \otimes \mathbb{C}_{\chi_{\Lambda}^{*}}$, viewed as module of the Levi factor $L_{S}=P_{S} \cap P_{S}^{o p p}$, is the trivial $L_{S}$-module. We thus obtain a well defined map

$$
\Gamma\left(G \times{ }_{P_{S}} \mathbb{C}_{\chi_{\Lambda}}\right) \times \Gamma\left(G \times{ }_{P_{S}^{o p p}} \mathbb{C}_{\chi_{\Lambda}^{*}}\right) \rightarrow \Gamma\left(G / L_{S}\right)
$$

(multiplication map), where $\Gamma\left(G / L_{S}\right)$ is the space of sections of the trivial line bundle over $G / L_{S}$. Hence the classical analogue of the algebra $\mathbb{A}_{\Lambda}$ is contained in the algebra of regular functions on $G / L_{S}$. The corresponding statement in the quantum setup is the inclusion $\mathbb{A}_{\Lambda} \subseteq \mathbb{C}_{q}\left[U / K_{S}\right]$, which follows from $[8$, Lem. 4.4]. In this note we prove the following stronger assertion.

Theorem 2.5. $\mathbb{A}_{\Lambda}=\mathbb{C}_{q}\left[U / K_{S}\right]$. 
In other words, a polynomial expression in the holomorphic and antiholomorphic quantum Plücker coordinates on $F_{\Lambda}$ is a quantum regular function on $U / K_{S}$ if and only if it has zero weight with respect to the left regular $U_{q}(\mathfrak{h})$ action. Furthermore, any quantum regular function on $U / K_{S}$ can be written in this form.

Remark 2.6. In [8] an (a priori) larger $*$-algebra $\mathbb{A}_{S} \supseteq \mathbb{A}_{\Lambda}$ was studied, called the factorized $*$-algebra. By definition, $\mathbb{A}_{S}$ is the span of the matrix coefficients $f_{\lambda} \cdot g_{\lambda}^{*}\left(f, g \in V(\lambda)^{*}\right)$ for all dominant weights $\lambda \in P^{+}$supported on $\Sigma \backslash S$. It was shown in $\left[8\right.$, Lem. 4.4] that $\mathbb{A}_{S} \subseteq \mathbb{C}_{q}\left[U / K_{S}\right]$. Thus Theorem 2.5 implies $\mathbb{A}_{S}=\mathbb{C}_{q}\left[U / K_{S}\right]$, which was conjectured in [8, Conj. 4.6]. For a special class of flag manifolds $U / K_{S}$ the equality $\mathbb{A}_{S}=\mathbb{C}_{q}\left[U / K_{S}\right]$ has been proved by a detailed analysis of the branching rules for certain finite dimensional $\mathfrak{g}$-representations (see [8, Thm. 4.10]). This class consists of flag manifolds $U / K_{S}$ for which $\mathfrak{p}_{S} \subset \mathfrak{g}$ is maximally parabolic and $\left(U, K_{S}\right)$ is a so-called Gel'fand pair (the most important examples are the compact Hermitean symmetric spaces, see $[8$, Prop. 4.7]).

The strategy for the proof of Theorem 2.5 is as follows. We first show that if suitable $C^{*}$-algebra completions of $\mathbb{A}_{\Lambda}$ and $\mathbb{C}_{q}\left[U / K_{S}\right]$ are the same, then automatically $\mathbb{A}_{\Lambda}=\mathbb{C}_{q}\left[U / K_{S}\right]$. Their $C^{*}$-algebra completions are shown to coincide by invoking a version of the Stone-Weierstrass Theorem for type I $C^{*}$-algebras. For the application of the Stone-Weierstrass type Theorem a detailed analysis of the irreducible $*$-representations of the two $C^{*}$-algebras is necessary, for which we can resort to (slight modifications of) the results in [8].

2.4. Towards a proof of Theorem 2.5. The normalized Haar functional on $\mathbb{C}_{q}[U]$ is the linear functional $h: \mathbb{C}_{q}[U] \rightarrow \mathbb{C}$ satisfying $h(1)=1$ (normalization) and satisfying $h \equiv 0$ on $W(\lambda)$ when $\lambda \in P^{+} \backslash\{0\}$. Equivalently, $h$ is the unique normalized linear functional on $\mathbb{C}_{q}[U]$ satisfying the biinvariance properties

$$
(h \otimes \mathrm{id}) \Delta(a)=h(a) 1=(\mathrm{id} \otimes h) \Delta(a), \quad \forall a \in \mathbb{C}_{q}[U] .
$$

A detailed study of Haar functionals in the algebraic framework was undertaken by Dijkhuizen and Koornwinder [1]. They consider a special class of Hopf-*algebras called compact quantum group algebras, a class containing $\mathbb{C}_{q}[U]$ as main example. In particular, application of $\left[1\right.$, Thm. 3.7] to $\mathbb{C}_{q}[U]$ implies that

$$
\langle a, b\rangle_{h}=h\left(b^{*} a\right), \quad \forall a, b \in \mathbb{C}_{q}[U]
$$

defines a pre-Hilbert structure on $\mathbb{C}_{q}[U]$. Let $\|\cdot\|_{h}$ be the corresponding norm on $\mathbb{C}_{q}[U]$.

Lemma 2.7. Let $A \subseteq B \subseteq \mathbb{C}_{q}[U]$ be subspaces invariant under the right regular $U_{q}(\mathfrak{g})$-action. If $A$ is dense in $B$ with respect to the norm $\|\cdot\|_{h}$, then $A=B$.

Proof. Let $A$ and $B$ be as in the lemma, and suppose that $A \subseteq B$ is dense but $A \neq B$. The Peter-Weyl decomposition (2.1) is the isotypical decomposition of 
$\mathbb{C}_{q}[U]$ under the right regular $U_{q}(\mathfrak{g})$-action, hence

$$
A=\bigoplus_{\lambda \in P^{+}} A(\lambda), \quad B=\bigoplus_{\lambda \in P^{+}} B(\lambda),
$$

with $A(\lambda)=A \cap W(\lambda)$ and $B(\lambda)=B \cap W(\lambda)$. The subspaces $A(\lambda)$ and $B(\lambda)$ $\left(\lambda \in P^{+}\right)$are finite dimensional. Hence, for some dominant weight $\mu \in P^{+}$, there exists a nonzero vector $b \in B(\mu)$ which is orthogonal to $A(\mu)$. We now use [1, Prop. 3.4], which asserts that the Peter-Weyl decomposition (2.1) is a decomposition in orthogonal subspaces. This implies that $b$ is orthogonal to $A$, contradicting the assumption that $A \subseteq B$ is dense.

Let $A$ be a unital $*$-algebra. Let $\mathcal{L}(H)$ be the $C^{*}$-algebra of bounded linear operators on the Hilbert space $H$. A unit preserving $*$-algebra homomorphism $\pi: A \rightarrow \mathcal{L}(H)$ is called a $*$-representation of $A$. We say that a $*$-representation $(\pi, H)$ of $A$ is irreducible if $H$ and $\{0\}$ are the only $\pi(A)$-invariant closed subspaces of $H$. Two $*$-representations $(\pi, H)$ and $\left(\pi^{\prime}, H^{\prime}\right)$ of $A$ are said to be equivalent if there exists an isomorphism $U: H \rightarrow H^{\prime}$ of Hilbert spaces intertwining the two actions of $A$.

By e.g. $[1, \S 4]$, we can now define a $C^{*}$-seminorm on the $*$-algebra $\mathbb{C}_{q}[U]$ by

$$
\|a\|_{\infty}=\sup _{\pi}(\|\pi(a)\|), \quad a \in \mathbb{C}_{q}[U],
$$

with $\pi$ running through a complete set of representatives of the irreducible $*-$ representations of $\mathbb{C}_{q}[U]$. The supremum in (2.2) may as well be taken over all *-representations $\pi$ of $\mathbb{C}_{q}[U]$.

It turns out that $\|\cdot\|_{\infty}$ is a $C^{*}$-norm on $\mathbb{C}_{q}[U]$. For this it suffices to construct a faithful $*$-representation of $\mathbb{C}_{q}[U]$. Such a faithful $*$-representation can be realized on the Hilbert space completion $\mathcal{H}_{h}$ of the pre-Hilbert space $\left(\mathbb{C}_{q}[U]\right.$, $\left.\langle\cdot, \cdot\rangle_{h}\right)$ by continuous extension of the regular $\mathbb{C}_{q}[U]$-representation

$$
\pi_{h}(a) b=a b, \quad \forall a, b \in \mathbb{C}_{q}[U],
$$

see [1, Lem. $4.2 \&$ Thm. 4.4].

Lemma 2.8. For all $a \in \mathbb{C}_{q}[U]$, we have $\|a\|_{h} \leq\|a\|_{\infty}$.

Proof. Observe that $\|1\|_{h}=1$ since the Haar functional $h$ is normalized, and that $\left\|\pi_{h}(a)\right\| \leq\|a\|_{\infty}$ for all $a \in \mathbb{C}_{q}[U]$. It follows that

$$
\|a\|_{h}=\left\|\pi_{h}(a) 1\right\|_{h} \leq\left\|\pi_{h}(a)\right\| \leq\|a\|_{\infty}
$$

for all $a \in \mathbb{C}_{q}[U]$.

The following corollary is a direct consequence of Lemma 2.7 and Lemma 2.8.

Corollary 2.9. Let $A \subseteq B \subseteq \mathbb{C}_{q}[U]$ be subspaces invariant under the right regular $U_{q}(\mathfrak{g})$-action. If $A$ is dense in $B$ with respect to the $C^{*}$-norm $\|\cdot\|_{\infty}$, then $A=B$. 
The completion $C_{q}(U)$ of $\mathbb{C}_{q}[U]$ with respect to the $C^{*}$-norm $\|\cdot\|_{\infty}$ is a unital $C^{*}$-algebra. The $C^{*}$-subalgebras $\mathcal{A}_{\Lambda} \subseteq C_{q}(U)$ and $C_{q}\left(U / K_{S}\right) \subseteq C_{q}(U)$ are defined to be the closures of $\mathbb{A}_{\Lambda}$ and $\mathbb{C}_{q}\left[U / K_{S}\right]$ in $C_{q}(U)$, respectively. Since $\mathbb{A}_{\Lambda} \subseteq \mathbb{C}_{q}\left[U / K_{S}\right]$, we have the inclusion $\mathcal{A}_{\Lambda} \subseteq C_{q}\left(U / K_{S}\right)$. The previous corollary implies:

Corollary 2.10. If $\mathcal{A}_{\Lambda}=C_{q}\left(U / K_{S}\right)$, then $\mathbb{A}_{\Lambda}=\mathbb{C}_{q}\left[U / K_{S}\right]$.

By Corollary 2.10 we can resort to the theory of $C^{*}$-algebras for the proof of Theorem 2.5. We first recall some general definitions and facts on $C^{*}$-algebras, starting with the notion of richness, cf. [2, Def. 11.1.1].

Definition 2.11. Let $B$ be a $C^{*}$-algebra. An $C^{*}$-subalgebra $A \subseteq B$ is called rich in $B$ if the following two conditions are satisfied:

- If $\pi$ is an irreducible $*$-representation of $B$, then its restriction $\left.\pi\right|_{A}$ is an irreducible $*$-representation of $A$.

- If $\pi$ and $\pi^{\prime}$ are two inequivalent irreducible $*$-representations of $B$, then $\left.\pi\right|_{A}$ and $\left.\pi^{\prime}\right|_{A}$ are inequivalent.

If $A \subseteq B$ is an inclusion of $*$-algebras, then we denote the restriction $\left.\pi\right|_{A}$ : $A \rightarrow \mathcal{L}(H)$ of a $*$-representation $\pi: B \rightarrow \mathcal{L}(H)$ again by $\pi$ if no confusion is possible.

We need the following analogue of the Stone-Weierstrass Theorem (see $[2$, Prop. 11.1.6]).

Theorem 2.12. Let $B$ be a type $I C^{*}$-algebra. The only rich $C^{*}$-subalgebra of $B$ is $B$ itself.

We apply this theorem to the inclusion $\mathcal{A}_{\Lambda} \subseteq C_{q}\left(U / K_{S}\right)$ of $C^{*}$-algebras. We start with the following elementary lemma.

Lemma 2.13. The $C^{*}$-algebra $C_{q}\left(U / K_{S}\right)$ is of type $I$.

Proof. By [6, Rem. 5.5], $C_{q}(U)$ is of type I. Now use that a $C^{*}$-subalgebra of a type I $C^{*}$-algebra is again of type I, see [2].

Lemma 2.14. The $C^{*}$-algebra $\mathcal{A}_{\Lambda}$ is rich in $C_{q}\left(U / K_{S}\right)$.

Observe that Theorem 2.5 is a direct consequence of Lemma 2.14. Indeed, Theorem 2.12, Lemma 2.13 and Lemma 2.14 imply $\mathcal{A}_{\Lambda}=C_{q}\left(U / K_{S}\right)$, whence $\mathbb{A}_{\Lambda}=\mathbb{C}_{q}\left[U / K_{S}\right]$ by Corollary 2.10 .

The proof of Lemma 2.14, which requires a detailed study of the irreducible *-representations of both the $C^{*}$-algebras $\mathcal{A}_{\Lambda}$ and $C_{q}\left(U / K_{S}\right)$, is given in the next section.

\section{Irreducible $*$-representations}

In this section we discuss the representation theory of the $*$-algebras $\mathbb{C}_{q}[U]$, $\mathbb{A}_{\Lambda}, \mathbb{C}_{q}\left[U / K_{S}\right]$ and their completions. 
3.1. The fundamental $*$-representation of $\mathbb{C}_{q}[\mathbf{S U}(2)]$. Let $V\left(=V\left(\varpi_{1}\right)\right)$ be the vector representation of $U_{q}(\operatorname{sl}(2, \mathbb{C}))$. Choose an inner product $(\cdot, \cdot)$ on $V$ such that $(X \cdot v, w)=\left(v, X^{*} \cdot w\right)$ for all $X \in U_{q}(\operatorname{sl}(2, \mathbb{C}))$ and all $v, w \in V$. Choose an orthonormal basis $\left\{e_{+}, e_{-}\right\}$of $V$ such that $e_{+}$(respectively $e_{-}$) is a highest (respectively lowest) weight vector of $V$. Then the four matrix coefficients

$$
L_{\epsilon \xi}=\left(\cdot e_{\xi}, e_{\epsilon}\right), \quad \epsilon, \xi= \pm
$$

are algebraic generators of $\mathbb{C}_{q}[\mathrm{SU}(2)]$. The Hopf- $*$-algebra structure of $\mathbb{C}_{q}[\mathrm{SU}(2)]$ can be completely characterized in terms of these generators (see e.g. [8, §3]).

The presentation of $\mathbb{C}_{q}[\mathrm{SU}(2)]$ in terms of the generators $L_{\epsilon \xi}$ can be used to define explicit $*$-representations of $\mathbb{C}_{q}[\mathrm{SU}(2)]$. In particular, if we write $\left\{e_{j}\right\}_{j=0}^{\infty}$ for the standard orthonormal basis of the Hilbert space $l_{2}\left(\mathbb{Z}_{+}\right)$, then it follows that

$$
\begin{aligned}
& \pi_{q}\left(L_{++}\right) e_{j}=\sqrt{\left(1-q^{2 j}\right)} e_{j-1}, \quad \pi_{q}\left(L_{+-}\right) e_{j}=-q^{j+1} e_{j}, \\
& \pi_{q}\left(L_{-+}\right) e_{j}=q^{j} e_{j}, \quad \pi_{q}\left(L_{--}\right) e_{j}=\sqrt{\left(1-q^{2(j+1)}\right)} e_{j+1}
\end{aligned}
$$

(with the convention that $\pi_{q}\left(L_{++}\right) e_{0}=0$ ) defines an irreducible $*$-representation $\pi_{q}$ of $\mathbb{C}_{q}[\mathrm{SU}(2)]$ on $l_{2}\left(\mathbb{Z}_{+}\right)$(see e.g. [6] and references therein). Up to tensoring with one-dimensional $*$-representations, $\pi_{q}$ is the only infinite dimensional irreducible $*$-representation of $\mathbb{C}_{q}[\mathrm{SU}(2)]$, cf. Section 3.2.

3.2. Representations of $\mathbb{C}_{q}[U]$. For $i=1, \ldots, r$ let $\phi_{i}: U_{q_{i}}(\operatorname{sl}(2, \mathbb{C})) \rightarrow U_{q}(\mathfrak{g})$ be the Hopf- $*$-algebra embedding defined on the generators by $\phi_{i}\left(K_{1}^{ \pm 1}\right)=K_{i}^{ \pm 1}$ and $\phi_{i}\left(X_{1}^{ \pm}\right)=X_{i}^{ \pm}$. The dual map induces a surjective Hopf- $*$-algebra homomorphism $\phi_{i}^{*}: \mathbb{C}_{q}[U] \rightarrow \mathbb{C}_{q_{i}}[\mathrm{SU}(2)]$. We write $\pi_{i}=\pi_{q_{i}} \circ \phi_{i}^{*}$ for the corresponding lift of $\pi_{q_{i}}: \mathbb{C}_{q_{i}}[\mathrm{SU}(2)] \rightarrow \mathcal{L}\left(l_{2}\left(\mathbb{Z}_{+}\right)\right)$to an irreducible $*$-representation of $\mathbb{C}_{q}[U]$.

One-dimensional $*$-representations of $\mathbb{C}_{q}[U]$ can be explicitly constructed as follows. Let $T=\mathbb{T}^{\times r}$ be the standard $r$-dimensional compact torus, where $\mathbb{T}=$ $\{z \in \mathbb{C}|| z \mid=1\}$ is the unit circle in the complex plane. Denote $t^{\mu}=t_{1}^{m_{1}} \cdots t_{r}^{m_{r}}$ for $\mu=\sum_{i=1}^{r} m_{i} \varpi_{i} \in P$ and $t=\left(t_{1}, \ldots, t_{r}\right) \in T$. Then an one-dimensional $*-$ representation $\tau_{t}: \mathbb{C}_{q}[U] \rightarrow \mathbb{C}(t \in T)$ can uniquely be defined by requiring that $\tau_{t}(a)=a(1) t^{\mu}$ when the quantum regular function $a \in \mathbb{C}_{q}[U]$ has left regular $U_{q}(\mathfrak{h})$-weight $\mu \in P$ (i.e. $K_{i} \cdot a=q^{\left(\mu, \alpha_{i}\right)} a$ for all $i$ ).

Let $\Delta^{(k)}: \mathbb{C}_{q}[U] \rightarrow \mathbb{C}_{q}[U]^{\otimes(k+1)}$ for $k \in \mathbb{Z}_{+}$be the iterated coproduct, defined recursively by $\Delta^{(0)}=\mathrm{id}$ and $\Delta^{(k+1)}=\left(\Delta \otimes \mathrm{id}^{\otimes k}\right) \circ \Delta^{(k)}$.

Theorem 3.1 (Soibel'man [6]). Let $w=s_{i_{1}} s_{i_{2}} \cdots s_{i_{l}}$ be a reduced expression for the Weyl group element $w \in W$, so that $l=l(w)$ is the length of $w$. Then the $*$-representation

$$
\pi_{w}=\left(\pi_{i_{1}} \otimes \pi_{i_{2}} \otimes \cdots \otimes \pi_{i_{l}}\right) \circ \Delta^{(l-1)}: \mathbb{C}_{q}[U] \rightarrow \mathcal{L}\left(l_{2}\left(\mathbb{Z}_{+}\right)^{\otimes l}\right)
$$

is independent of the reduced expression (up to equivalence), and

$$
\left\{\pi_{w, t}=\left(\pi_{w} \otimes \tau_{t}\right) \circ \Delta \mid w \in W, t \in T\right\}
$$

is a complete set of mutually inequivalent irreducible $*$-representations of $\mathbb{C}_{q}[U]$. 
Remark 3.2. An important role in the proof of Theorem 3.1 is played by $[6$, Thm. 3.1], which states that $\mathbb{C}_{q}[U]$ is algebraically generated by the quantum regular functions $f_{\lambda}$ and $g_{\lambda}^{*}$ for all $f, g \in V(\lambda)$ and all $\lambda \in P^{+}$. The proof of [6, Thm. 3.1] uses properties of the branching rules for tensor products of finite dimensional irreducible $\mathfrak{g}$-modules when the highest weights become large, see e.g. [3, Prop. 9.2.2]. Theorem 2.5 should be viewed as the analogue of [6, Thm. 3.1] for the generalized flag manifold $U / K_{S}$. The analogous statement for the Poisson homogeneous space $U / K_{S}^{0}$ is formulated in Theorem 4.1(a).

3.3. Representations of $\mathcal{A}_{\Lambda}$. The $*$-algebra $\mathbb{A}_{\Lambda}$ has the convenient property that it is defined in terms of explicit algebraic generators involving quantum Plücker coordinates. This property enables one to analyze the *-representations of $\mathbb{A}_{\Lambda}$ in a similar manner as for $\mathbb{C}_{q}[U]$, cf. Remark 3.2. This analysis was carried out in $[8, \S 6]$, but then for the a priori larger $*$-algebra $\mathbb{A}_{S}$ (see Remark 2.6). Straightforward adjustments of the proof of [8, Thm 6.13] though show that $\left\{\left(\pi_{w}, l_{2}\left(\mathbb{Z}_{+}\right)^{\otimes l(w)}\right) \mid w \in W^{S}\right\}$ is a complete set of mutually inequivalent irreducible $*$-representations of $\mathbb{A}_{\Lambda}$.

The irreducible $*$-representation $\pi_{w}\left(w \in W^{S}\right)$ of $\mathbb{A}_{\Lambda}$ is the restriction to $\mathbb{A}_{\Lambda}$ of an $*$-representation of $\mathbb{C}_{q}[U]$. Hence it extends by continuity to an irreducible *-representation of $\mathcal{A}_{\Lambda}$, which we again denote by $\pi_{w}$. On the other hand, any irreducible $*$-representation of $\mathcal{A}_{\Lambda}$ restricts to an irreducible $*$-representation of $\mathbb{A}_{\Lambda}$. We conclude that:

Corollary 3.3. $\left\{\pi_{w} \mid w \in W^{S}\right\}$ is a complete set of mutually inequivalent irreducible *-representations of the $C^{*}$-algebra $\mathcal{A}_{\Lambda}$.

3.4. Representations of $\mathbb{C}_{q}\left[U / K_{S}\right]$. In this section we first establish the analogue of Corollary 3.3 for $C_{q}\left(U / K_{S}\right)$. Since we (a priori) do not have a complete set of algebraic generators of the $*$-algebra $\mathbb{C}_{q}\left[U / K_{S}\right]$, we cannot analyze its *-representations in the same manner as for $\mathbb{A}_{\Lambda}$. The alternative approach is by analyzing the ireducible $*$-representations of the $C^{*}$-algebra $C_{q}\left(U / K_{S}\right)$ directly. For this, we first observe that the inclusion $\mathbb{A}_{\Lambda} \subseteq \mathbb{C}_{q}\left[U / K_{S}\right]$ and the results in Section 3.3 imply that $\pi_{w}\left(w \in W^{S}\right)$ are mutually inequivalent irreducible $*-$ representations of $\mathbb{C}_{q}\left[U / K_{S}\right]$ and of $C_{q}\left(U / K_{S}\right)$. Furthermore, by [8, Prop. 5.7] we have

$$
\pi_{w, t}(a)=\pi_{u}(a) \otimes \mathrm{id}^{\otimes l(v)}, \quad \forall a \in C_{q}\left(U / K_{S}\right)
$$

for $t \in T$ and $w=u v \in W$ with $u \in W^{S}$ and $v \in W_{S}$. Since every irreducible *-representation of $C_{q}\left(U / K_{S}\right)$ appears as irreducible component of $\left.\pi\right|_{C_{q}\left(U / K_{S}\right)}$ for some irreducible $*$-representation $\pi$ of $C_{q}(U)$ (see e.g. [2, Prop. 2.10.2]), we conclude (cf. [8, Thm. 5.9]):

Corollary 3.4. $\left\{\pi_{w} \mid w \in W^{S}\right\}$ is a complete set of mutually inequivalent irreducible *-representations of the $C^{*}$-algebra $C_{q}\left(U / K_{S}\right)$.

Corollary 3.3 and Corollary 3.4 show that $\mathcal{A}_{\Lambda}$ is rich in $C_{q}\left(U / K_{S}\right)$. Thus Lemma 2.14 is proved, which in turn implies the validity of Theorem 2.5 (see Section 2.4). 
In particular, the classification of the irreducible $*$-representations of $\mathbb{A}_{\Lambda}$ (see Section 3.3) gives the following theorem.

Theorem 3.5. The set

$$
\left\{\left(\pi_{w}, l_{2}\left(\mathbb{Z}_{+}\right)^{\otimes l(w)}\right) \mid w \in W^{S}\right\}
$$

is a complete set of mutually inequivalent irreducible *-representations of the quantized function algebra $\mathbb{C}_{q}\left[U / K_{S}\right]$.

\section{Remark 3.6.}

(a) Theorem 3.5 is not a direct consequence of Corollary 3.4, since it is a priori not clear that every irreducible $*$-representation of $\mathbb{C}_{q}\left[U / K_{S}\right]$ can be continuously extended to a $*$-representation of $C_{q}\left(U / K_{S}\right)$.

(b) Theorem 3.5 fits nicely in the philosophy of the Kostant-Kirillov orbit method. Indeed, the irreducible $*$-representations of $\mathbb{C}_{q}\left[U / K_{S}\right]$ are parametrized by the coset representatives $W / W_{S}$, which in turn also parametrize the symplectic leaves of the underlying Poisson $U$-homogeneous space $U / K_{S}$, see Section 2.1 and $[8, \S 2]$.

\section{The Poisson $U$-homogeneous space $U / K_{S}^{0}$.}

In this section we apply the methods of the present note to the Poisson $U$ homogeneous space $U / K_{S}^{0}$.

Denote $U_{q}\left(\mathfrak{k}_{S}^{0}\right)$ for the unital Hopf- $*$-subalgebra of $U_{q}(\mathfrak{g})$ generated by $K_{i}^{ \pm 1}, X_{i}^{ \pm}$ $(i \in S)$. We call

$$
\mathbb{C}_{q}\left[U / K_{S}^{0}\right]=\left\{a \in \mathbb{C}_{q}[U] \mid X \cdot a=\epsilon(X) a, \forall X \in U_{q}\left(\mathfrak{k}_{S}^{0}\right)\right\} \subseteq \mathbb{C}_{q}[U]
$$

the $*$-algebra of quantized regular functions on the Poisson $U$-homogeneous space $U / K_{S}^{0}$. Note that $\mathbb{C}_{q}\left[U / K_{S}^{0}\right]$ is invariant under the right regular $U_{q}(\mathfrak{g})$-action and under the left regular $U_{q}(\mathfrak{h})$-action. Observe furthermore that

$$
\mathbb{C}_{q}\left[U / K_{S}\right]=\left\{a \in \mathbb{C}_{q}\left[U / K_{S}^{0}\right] \mid X \cdot a=\epsilon(X) a \quad \forall X \in U_{q}(\mathfrak{h})\right\} .
$$

\section{Theorem 4.1.}

(a) $\mathbb{C}_{q}\left[U / K_{S}^{0}\right]$ is algebraically generated by the matrix coefficients $f_{\varpi_{k}}$ and $g_{\varpi_{l}}^{*}$ for all $f \in V\left(\varpi_{k}\right)^{*}, g \in V\left(\varpi_{l}\right)^{*}$ and all $k, l \in \Sigma \backslash S$.

(b) A complete set of mutually inequivalent irreducible $*$-representations of the *-algebra $\mathbb{C}_{q}\left[U / K_{S}^{0}\right]$ is given by

$$
\left\{\left(\pi_{w, t}, l_{2}\left(\mathbb{Z}_{+}\right)^{\otimes l(w)}\right) \mid w \in W^{S}, t \in T_{\Sigma \backslash S}\right\},
$$

with $T_{\Sigma \backslash S} \subseteq T$ the sub-torus

$$
T_{\Sigma \backslash S}=\left\{t=\left(t_{1}, \ldots, t_{r}\right) \in T \mid t_{i}=1 \text { when } i \in S\right\} .
$$




\section{Remark 4.2.}

(a) The special case $S=\emptyset$ of Theorem 4.1 recovers Soibel'man's [6] results for $\mathbb{C}_{q}[U]$. The irreducible $*$-representations for the special case that $U / K_{S}^{0}$ is the Stiefel manifold $\mathrm{SU}(n) / \mathrm{SU}(l)(l<n)$ were described before by Podkolzin and Vainerman [5].

(b) The unital $*$-algebra $\mathbb{A}_{\Lambda}^{0}$ algebraically generated by the holomorphic and antiholomorphic quantum Plücker coordinates $f_{\Lambda}$ and $g_{\Lambda}^{*}(f, g \in \mathcal{B})$ is in general properly contained in $\mathbb{C}_{q}\left[U / K_{S}^{0}\right]$. This can for instance be verified by comparing the weights occurring in $\mathbb{A}_{\Lambda}^{0}$ and in $\mathbb{C}_{q}\left[U / K_{S}^{0}\right]$ under the left regular $U_{q}(\mathfrak{h})$-action.

\section{Acknowledgements}

The author is supported by a fellowship from the Royal Netherlands Academy of Arts and Sciences (KNAW).

\section{References}

[1] M.S. Dijkhuizen, T.H. Koornwinder, CQG algebras: a direct approach to compact quantum groups, Lett. Math. Phys. 32 (1994), 315-330.

[2] J. Dixmier, $C^{*}$-algebras, North-Holland Mathematical Library, Vol. 15. North-Holland Publishing Co., Amsterdam-New York-Oxford, 1977.

[3] A. Joseph, Quantum groups and their primitive ideals, Ergebnisse der Mathematik und ihrer Grenzgebiete (3), 29. Springer-Verlag, Berlin, 1995.

[4] J.-H. Lu, A. Weinstein, Poisson-Lie groups, dressing transformations, and Bruhat decompositions, J. Differential Geom. 31 (1990), 501-526.

[5] G. B. Podkolzin, L. I. Vainerman, Quantum Stiefel manifold and double cosets of quantum unitary group, Pacific J. Math. 188 (1999), 179-199.

[6] Ya.S. Soibel'man, Algebra of functions on a compact quantum group, and its representations, Leningrad Math. J. 2 (1991), 161-178.

[7] - On the quantum flag manifold, Funct. Anal. Appl. 26 (1992), 225-227.

[8] J.V. Stokman, M.S. Dijkhuizen, Quantized flag manifolds and irreducible *representations, Comm. Math. Phys. 203 (1999), 297-324.

KdV Institute for Mathematics, Universiteit van Amsterdam, Plantage MuiderGracht 24, 1018 TV Amsterdam, The Netherlands.

E-mail address: jstokman@science.uva.nl 